Marquette University

e-Publications@Marquette

5-8-2006

\title{
Micromagnetic Simulations on the Dependence of Gilbert Damping on Domain Wall Velocities in Magnetic Nanowires
}

Andrew Kunz

Marquette University, andrew.kunz@marquette.edu

B. Kastor

Marquette University

Accepted version. Published as a part of the proceedings of the 2006 IEEE International Magnetics Conference (INTERMAG), DOI. (C) 2006 IEEE. Used with permission. 


\title{
e-Publications@Marquette
}

\section{Physics Faculty Research and Publications/College of Arts and Sciences}

This paper is NOT THE PUBLISHED VERSION; but the author's final, peer-reviewed manuscript. The published version may be accessed by following the link in th citation below.

2006 IEEE International Magnetics Conference (INTERMAG), (May 8-12, 2006). DOI. This article is (C) Institute of Electrical and Electronic Engineers (IEEE) and permission has been granted for this version to appear in e-Publications@Marquette. Institute of Electrical and Electronic Engineers (IEEE) does not grant permission for this article to be further copied/distributed or hosted elsewhere without the express permission from Institute of Electrical and Electronic Engineers (IEEE).

\section{Micromagnetic Simulations on the Dependence of Gilbert Damping on Domain Wall Velocities in Magnetic Nanowires}

\author{
A. Kunz \\ Physics, Marquette University, Milwaukee, WI \\ B. Kastor \\ Physics, Marquette University, Milwaukee, WI
}

\begin{abstract}
:
The dependence of damping on domain wall motion and velocity in Permalloy nanowires is presented. The domain wall motion in isolated two micron long Permalloy nanowires, with a rectangular cross-section 10 $\mathrm{nm}$ thick and $100 \mathrm{~nm}$ wide, is simulated using the Landau-Lifshitz Gilbert (LLG) simulation.Interpreting LLG dynamics can be difficult due to the dependence of the results on the Gilbert damping parameter alpha. The Walker model also predicts the critical field and domain wall velocity as a function of alpha. For these combined reasons the dependence of the domain wall speeds on the damping parameter is explored.
\end{abstract}

The dependence of damping on domain wall motion and velocity in Permalloy nanowires is presented. Magnetic nanowires have potential for application in many technological devices, but to be useful domain walls must move reliably and quickly along the wire. It has recently been shown that domain wall velocities in nanowires are significantly slower than in bulk materials, and slower than predicted by current analytic 
models [1], [2]. It has also been shown that an increase in the driving field can lead to a further reduction in the domain wall speed [2], [3]. The reduced velocity is due to the nucleation of vortices in the domain wall. This behavior is called Walker breakdown, and the driving field at which the nucleation appears is called the Walker field [4]. Due to the difficulty of obtaining high-resolution imaging of domain walls in motion with in a nanowire, micromagnetic simulation is used to give insight into the mechanisms limiting domain wall speeds.

The domain wall motion in isolated two micron long Permalloy nanowires, with a rectangular cross-section $10 \mathrm{~nm}$ thick and $100 \mathrm{~nm}$ wide, has been simulated using the Landau-Lifshitz Gilbert (LLG) simulation [5]. Interpreting LLG dynamics can be difficult due to the dependence of the results on the Gilbert damping parameter alpha. The Walker model also predicts the critical field and domain wall velocity as a function of alpha. For these combined reasons the dependence of the domain wall speeds on the damping parameter is explored in this paper.

The domain wall dynamics are explored for fields above, at, and below the critical Walker field for a range of damping value found in experiment. As has been previously reported, below the Walker field the domain wall velocity increases as the applied field is increased [2], [3]. This is because the domain wall moves uniformly down the length of the wire. Above the Walker field, magnetic vortices are nucleated in the wire and the domain wall motion is suppressed. The nucleation process significantly lowers the value of the domain wall velocity. The results are quantified by looking at the velocity. These results are summarized in figure 1 show the average domain wall speed along the wire as a function of the applied field for two values of the damping parameter alpha. The significant drop in the domain wall velocity is due to the nucleation of magnetic vortices in the domain wall. The process leading to the nucleation of the vortices in the domain wall above the critical field will be presented. The inset of figure one shows the domain wall velocity when driven along the wire by a 100 Oe field for alpha values over two orders of magnitude $(0.001<\alpha<0.1)$. The results show that by increasing the value of alpha the domain wall velocity increases.

The Walker model significantly overestimates the critical field and domain wall velocity in nanowires. The Walker model is based on bulk magnetic properties, the results of which do not hold on the reduced dimensions found in the nanowires. The analytic Walker model will be discussed, and the simulation results will be compared to the current Walker model. The results of the simulations will be used to improvements to the analytic model necessary to be valid in the reduced nanowire geometry.

The authors thank Dan Dahlberg, and the Marquette University Helen Way Klingler College of Arts and Science for their support of this project. 


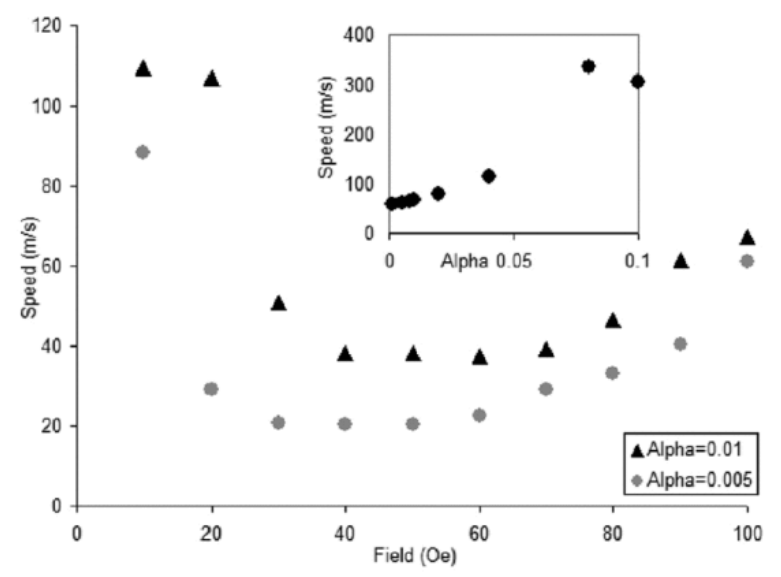

Fig. 1: Average domain wall velocities in a Permalloy nanowire as a function of driving field for two values of the damping parameter alpha. The drop in speed is due to the nucleation of vortices in the domain wall. The inset shows how the wall speed increases with damping.

\section{References}

1. Y. Nakatani, A. Thiaville, J. Miltat, "Faster magnetic walls in rough wires", Nature Materials, vol. 2, pp. 521-523, 2003.

2. A. Kunz, "Simulated domain wall dynamics in magnetic nanowires", J. Appl. Phys., June 2006.

3. A. Yamaguchi et al., "Real-space observation of current-driven domain wall motion in submicron magnetic wires", Phys. Rev. Lett., vol. 92, pp. 077205, 2004.

4. N.L. Schryer, L.R. Walker, "The motion of $180^{\circ}$ domain walls in uniform dc magnetic fields", J. Appl. Phys, vol. 45, pp. 5406-5421, 1974.

5. Tempe, AZ 85282, USA:LLG Inc. 